\title{
Functionally Substituted Chemical Organic Compounds: Potential Antimicrobial Substances
}

\section{Shoailb M and Ganbarov G Kh* \\ Department of Microbiology, Faculty of Biology, Baku State University, Azerbaijan}

*Corresponding author: Ganbarov Khudaverdi Ganbar, Head of Department of Microbiology, Faculty of Biology, Baku State University, Azerbaijan, Tel: +994503270556; Email: khuda1949@mail.ru

\section{Review Article \\ Volume 4 Issue 1}

Received Date: January 07, 2019

Published Date: January 28, 2019

DOI: $10.23880 /$ oajmb-16000136

\section{Abstract}

The development of resistance to antimicrobial agents is a global health problem for humans as well as animals. Over the past decade, the rate of microbial resistance to antibiotics has alarmingly increased. Factors contributing to this ever increasing antibiotic resistance pertain to microorganism as well as excessive and unwise use of the antibiotics. Biofilm formation and use of efflux pumps are the examples of microbial factors responsible for emergence of antimicrobial resistance. To ameliorate this condition, it is dire need of time to develop new antimicrobial agents. In this regard, chemical organic compounds are best available substances with potential antimicrobial properties. This review summarizes the antimicrobial activities of some important organic compounds as well as potential of functionally substituted chemical organic compounds as probable antimicrobial agents of future. These important organic compounds include functionally substituted derivatives of cyclohexane, quaternary ammonium compounds, hydrazones, pyrazolone, triazole, thiones, isatin, indole, chalcones, quinoxaline, anthraquinones, coumarins, thiophenes, piperidine, benzoxazolinone, phthalazinone, sulfones, thiazole and volatile organic compounds.

Keywords: Antimicrobial Resistance; Cyclohexane; Functionally Substituted Derivatives; Biofilm; Organic Compounds

\section{Introduction}

In the present time, drug development studies are of paramount importance because of increasing resistance of microbes to antibiotics and shortage of new antimicrobial drugs [1]. Bacteria have developed resistance against different groups of antibiotics like $\beta$ lactams, quinolones, vancomycin, glycopeptides, macrolides etc. Multi drug resistant and extensively drug resistant bacteria are leading cause of casualties due to infectious diseases [2]. Currently another group of bacteria called pan drug-resistant bacteria, which are resistant to almost all the available antibiotics has emerged [3]. In this regard many studies are being carried out round the world to unfold new possible antimicrobial compounds. Hybrid organic molecules, combining two or more potentially bioactive substructures to make an integrated new organic compound with a higher anticipated antimicrobial potential are currently the targets of the worldwide drug discovery studies [4]. In order to ameliorate the problem of drug resistance and synthesize new and better therapeutic agents, there is an urgent need to explore new chemical organic compounds with potential antimicrobial properties and unique mode of action which is not affected by bacterial resistance. The objective of this review is to provide recent insights and experimental evidences of antimicrobial potential of chemical compounds and their derivatives. 


\section{Open Access Journal of Microbiology \& Biotechnology}

\section{Mechanism of Action}

Many chemical organic compounds and their derivatives e.g. quaternary amine derivatives, functionally substituted cyclohexane, compounds with various alkyl chain lengths, alkyl chain with different hydrophobic substitutes etc are being considered and explored as potential antimicrobial agents [5]. Profound efforts are made to explore the mode of action of these potential antimicrobial compounds. For example, Quaternary Ammonium compounds have lethal effects on bacterial cells by affecting their cell walls [6]. Organic compounds having Quaternary Ammonium moiety inhibit the bacterial biofilm formation, which is one of major cause of bacterial resistance. These compounds induce apoptosis in bacterial cell by interacting with genomic material of bacterial cells and destroy cellular proteins of bacteria [3]. Heterocyclic pipyridinium and glucopyranose quaternary ammonium salts are new groups of Quaternary Ammonium compounds which have shown good antimicrobial activity [7]. Thienopyrimidines act on membrane potentials, membrane permeability and net surface charge of the bacterial membranes by their physicochemical characteristics leading to leakage of cellular contents and bacterial death [8]. Phenolic compounds are widely used as antibacterial agents and numerous mechanisms are understood for their bactericidal activity. Disruption of bacterial cytoplasmic membrane, action on bacterial nuclear material at chromosome level and coagulation of bacterial proteins are major mechanisms responsible for bactericidal activity of Phenolic compounds [9]. Synthetic tricyclic flavonoids are important class of organic compounds which possess bacteriostatic and bactericidal effects. Mode of action of these compounds is impairment of bacterial cell membrane and bacterial cell agglutination [10].

Schiff bases like Hydrazones are important group of organic compounds which show strong antimicrobial, anti inflammatory and anti viral effects [11]. Heterocyclic compounds containing thiones and pyrimidines have great pharmacological value because of wide range of biological activity. These compounds have great potential as antiviral, antibacterial, antifungal and anti inflammatory mediators [12]. Cyclohexane triones are synthetic antimicrobial compounds that show activity against gram-positive bacteria, Haemophilus influenzae and Mycobacterium smegmatis. In contrast to other cationic antibacterial agents which disrupt the bacterial cytoplasmic membrane, cyclohexane triones block the transport of low molecular weight substances into bacterial cells [13]. Pyrazolone derivatives have numerous applications in the medicine due to wide range of biological activities including antimicrobial and anti neoplastic activity. These compounds incorporated with sulfonyl phosphonates can act as potential antimicrobial compounds [14]. Phthalazinone is very important chemical which have found its application in clinical medicine due to wide range of biological effects. Many drug molecules like Hydralazine, Budralazine, Azelastine, Ponalrestat, Zopolrestat etc are derived starting from nucleus of Phthalazinone [15]. Thiazole derivatives are important organic compounds with antibacterial and antifungal activities. The structure of 1,3-thiazolidin-4one is so flexible that by inserting the substitutes into various positions of 1,3-thiazolidin-4-one ring, new biologically active compounds can be made. In thiazole ring, most active positions are 2,3 , and 5 , which play major role in directions of heterocyclic modification. At 5position of 4-thiazolidones, methylene group is more active, that's why usually substitutions are made at this position [16]. Nitrofurans possess broad spectrum antimicrobial properties but these are highly toxic compounds. By inserting Nitrofuran at 5-position of 4thiazolidone ring and allyl group into 3-position, new chemical compounds with high antimicrobial activity and less toxicity can be designed [17].

Isatin derivatives (indole-2,3-dione) and its Schiff and Mannich bases have broad range of biological activities including antibacterial, antifungal and anti HIV activities. Hence different derivatives of isatin like hydrazine derivatives, acetone and ketone derivatives, thiosemicarbazone derivates etc are being explored for their potential against tuberculosis, leprosy, fungal, viral and bacterial infections and Trypnosomiasis. Besides this, antioxidative activity of Isatin derivatives has also been reported [18].

\section{Antimicrobial Properties of Functionally Substituted Chemical Compounds}

Antimicrobial properties of forty two adamantyl based cyclohexane diamine derivatives (as shown in Figure 1) were examined against 29 strains of Methicillin resistant Staphylococcus aureus (MRSA) and a virulent strain of Mycobacterium tuberculosis. For these synthetic Cyclohexane derivatives, minimum inhibitory concentrations were determined. Against MRSA, strong to moderate antibacterial activity was observed while for Mycobacterium tuberculosis, moderate to weak antibacterial activity was observed. Some of these derivatives showed bactericidal effects against 


\section{Open Access Journal of Microbiology \& Biotechnology}

Mycobacterium tuberculosis with rapid kill kinetics showing $4 \log$ declines in viability of bacterial cells [19].

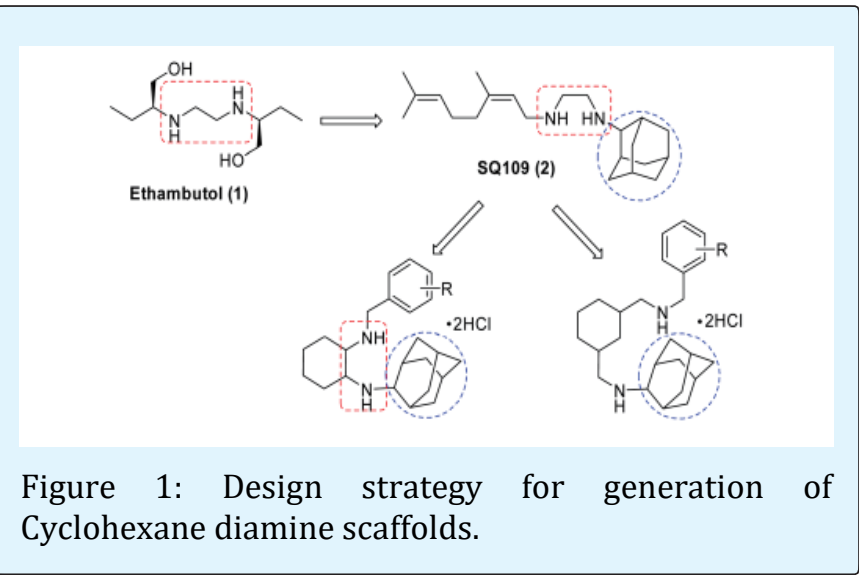

A new group of trisazo derivatives were prepared by combining the diazonium ion of 3-amino-4Hthieno [3,4-c] [1] benzopyran-4-one with 2tert-butyl-4-methoxyphenol. Using micro dilution susceptibility assay, the compound was screened for antimicrobial properties against Bacillus subtilis, Staphylococcus aureus, Escherichia coli, Pseudomonas aeruginosa, Vibrio cholerae, Candida parapsilosis, Candida albicans and Candida neoformans. The compound having molecular formula $\mathrm{C}_{44} \mathrm{H}_{38} \mathrm{~N}_{6} \mathrm{O}_{13} \mathrm{~S}_{3}$ was most active antimicrobial agent. It was concluded that Candida neoformans was most sensitive microorganism, while Vibrio cholerae was most resistant microorganism to trisazo derivatives [4]. Organic Functionalized Graphene-Oxide with Selected Amine derivatives (Figure 2) were characterized and evaluated for antimicrobial activities. These amines were screened for antibacterial potential against Escherichia coli, Pseudomonas aeruginosa and Staphylococcus aureus in both plankonic and biofilm growth state. Functionalized graphene oxide derivatives showed better antimicrobial activity against plankonic form while amine derivatives exhibited better antimicrobial activity against biofilm embedded cells [20].

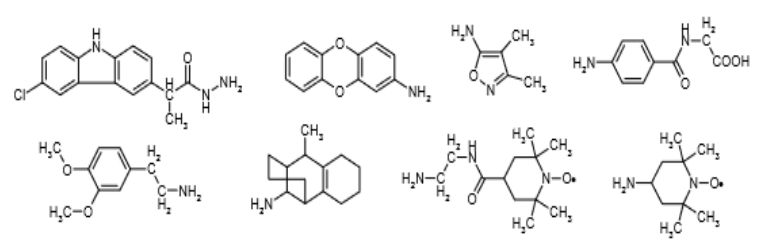

Figure 2: Chemical structure of Graphene-Oxide Amine derivatives.
A series of 11 thienopyrimidines derivatives were prepared and examined for antimicrobial properties against Staphylococcus aureus, Escherichia coli, Fusarium oxysporum and Candida albicans. Agar diffusion method was used for antimicrobial screening. Thienopyrimidines derivatives were effective against all the tested microorganisms except Fusarium oxysporum. The compound having Structural formula of 2-[2(diphenylmethylene) hydrazino]-5-isopropyl-3methylthieno [2, 3-d] pyrimidin-4-one showed most strong antimicrobial properties [8]. Six new benzoxazolinone derivatives were prepared and investigated for antimicrobial properties. Tube-dilution method and cylinder plate method were used to screen these compounds against Bacillus subtilis, Staphylococcus aureus, Escherichia coli and Candida albicans. Two derivatives shown in Figure 3 were found to be best antimicrobial and antifungal agents [21].

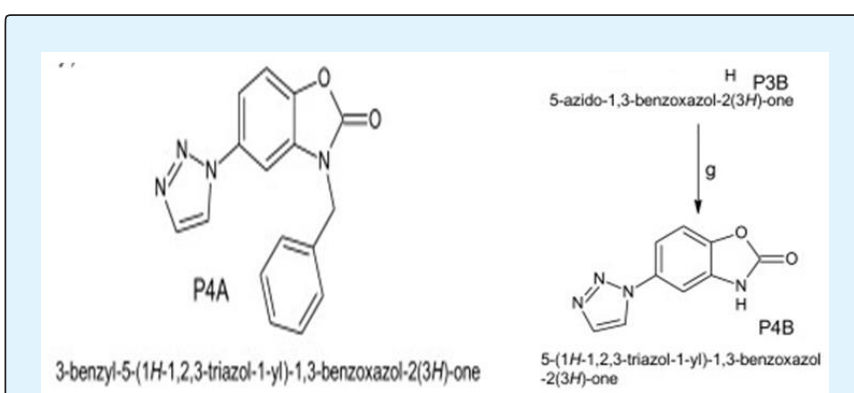

Figure 3: Benzoxazolinone derivatives with maximum antimicrobial potential.

The new functionally substituted benzylidenehydrazinyl pyridinium salts with the hydroxyl, methyl and methoxyl substituent in ortho position of benzene ring were synthesized and tested for antimicrobial activity against gram positive bacteria, gram negative bacteria and fungi. Using micro dilution assay, minimum inhibitory concentrations were determined against Staphylococcus aureus, Escherichia coli, Pseudomonas aeruginosa and Candida albicans. Maximum antimicrobial activity against Staphylococcus aureus was shown by compounds containing a 3phenylpropyl chain. Against all the tested strains of bacteria and fungi, 4-(2-(2-methylbenzylidene) hydrazinyl)-1-(3phenylpropyl) pyridinium bromide was most active antimicrobial agent. The longer side chain on pyridinium nitrogen is responsible for high antimicrobial activity [5]. A new class (twenty four compounds) of esters of 4-acetyl, 4-trifluoroacetyl- and 4-(3chloropropionyl) aminobenzenethiosulfoacids were synthesized and evaluated for antimicrobial properties. Using the agar diffusion and serial dilution methods, 


\section{Open Access Journal of Microbiology \& Biotechnology}

antimicrobial screening was performed against Staphylococcus aureus, Bacillus subtilis, Bacillus mesentericus, Mycobacterium sp., Mycobacterium luteum, Aeromonas sp., Burkholderia

cepacia, Alcaligenes faecalis, Pseudomonas aeruginosa, Escherichia coli, Proteus vulgaris, Candida albicans, Candida tenuis, Candida glabrata, Verticillium dahliae, Trichophyton gypseum, Aspergillus niger, Aspergillus fumigatus and Penicillium chrysogenum. All the tested compounds showed considerable antimicrobial properties but cycloalkyl and aryl esters of 4acylaminobenzenethiosulfoacids manifested strong antimicrobial activity at nano molar concentrations [22].

About 22 different $\alpha \mathrm{N}$-Phthilimido and acetylated derivatives of amino acids were prepared and tested for antimicrobial profile. Antibacterial screening was performed by disc diffusion method using Mueller-Hinton agar medium against Escherichia coli, Klebsiella, Staphylococcus epidermidis, Bacillus cereus, Micrococcus luteus and Staphylococcus aureus. Antifungal screening was done by using cup plate method on potato dextrose agar against Candida albicans, and Aspergillus niger. All the synthesized compounds showed considerable antimicrobial effect against all the tested microorganisms except for Candida albicans [23]. Novel heterocyclic compounds containing imidazole moiety from $\alpha$-oxoketen dithioacetals and thiocarbamyl were synthesized as potential therapeutic compounds. Antimicrobial properties of these compounds were evaluated against Staphylococcus aureus, Bacillus subtilis, Pseudomonas aeruginosa, Escherichia coli, Aspergillus fumigates, Syncephalastrum racemosum, Geotrichum candidum and Candida albicans. Ampicillin, gentamycin and amphotericin B were used as standard drugs for comparisons of antimicrobial properties of tested compounds. All the tested compounds showed better antibacterial activity against gram positive bacteria as compared to gram negative bacteria. 3,3-Bis(methylthio)2-[1-(4-chlorophenyl)-4,5-diphenyl-1H-imidazol-2ylthio]-N-p- tolylacrylamide was best antibacterial and antifungal agent as it exhibited strong antimicrobial properties than Ampicillin, gentamycin and amphotericin B [24].

A series of 3,4-dihydropyrimidine-2(1H)-thiones were investigated for antimicrobial properties against Staphylococcus aureus, Escherichia coli, Bacillus subtilis, Aspergillus niger and Penicillium species. Well diffusion method was used to determine the antibacterial and antifungal potential of thione derivatives. Only 6-(2,5Dichlorothiophen-3-yl)-4-(4-flourophenyl)-3,4-
dihydropyrimidine-2(1H)-thione showed considerable antimicrobial activity against Staphylococcus aureus, Bacillus subtilis and Aspergillus niger [12]. A new class of compounds containing chiral quaternary $\mathrm{N}$-spiro ammonium bromides with $3^{\prime}, 4^{\prime}$-dihydro- $\mathbf{1}^{\prime} \mathrm{h}$ spiro[isoindoline-2,2'isoquinoline] skeleton were synthesized and checked for antibacterial properties. Minimum inhibitory concentration and minimum bactericidal concentration values were determined against Staphylococcus aureus, Streptococcus pyogenes, Streptococcus mutans, Streptococcus salivarius, Bacillus subtilis, Enterococcus faecalis, Moraxella catarrhalis, Escherichia coli and Campylobacter jejuni by broth micro dilution method. All the tested compounds showed antibacterial activity and these compounds were more active against gram negative bacteria as compared to gram positive bacteria. Figure 4 shows the scheme of structure of compounds which showed maximum antibacterial activity [13].

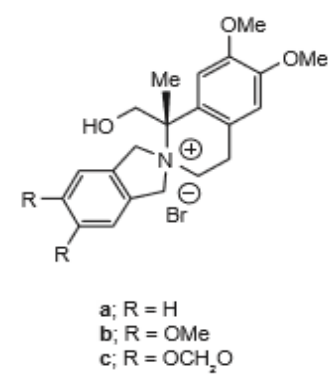

Figure 4: Structure of compounds which showed maximum antibacterial activity

Iron cyclohexanedicarboxylic acid was synthesized (Figure 5) and examined for antibacterial and antifungal properties. Using agar well diffusion susceptibility test, antibacterial properties were seen against Bacillus subtilis, Staphylococcus aureus, Escherichia coli and Pseudomonas aeruginosa. Using agar tube dilution protocol method, antifungal properties were determined against Candida albicans, Candida glabrata and Fusarium solani. Iron cyclohexanedicarboxylic acid was found to be effective against gram positive bacteria and fungi, but no antimicrobial activity was seen against gram negative bacteria [25].

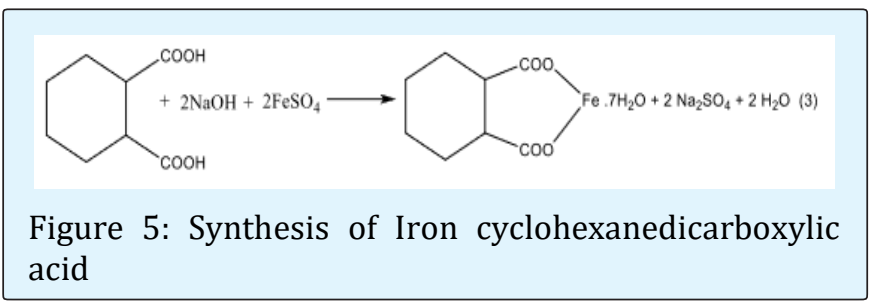




\section{Open Access Journal of Microbiology \& Biotechnology}

A series of 56 unsymmetrically substituted cyclohexane-1,2-diamine derivatives with different substitution pattern on the Benzene ring were prepared and their antimicrobial properties were tested against Escherichia coli, Pseudomonas aeruginosa, Staphylococcus aureus and Staphylococcus epidermidis. Minimum inhibitory concentration of these derivatives was determined against these pathogens and Tetracycline was used as standard drug for comparison. Cyclohexane derivatives with chloro, bromo or fluoro groups at para position of Benzene ring (Figure 6) showed stronger antimicrobial activities as compared to compounds in which these groups were present at meta position of Benzene ring [2].
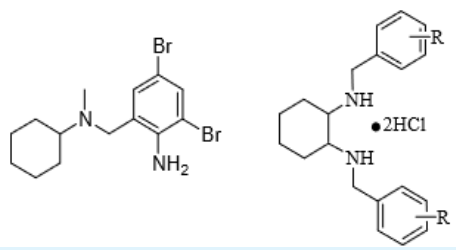

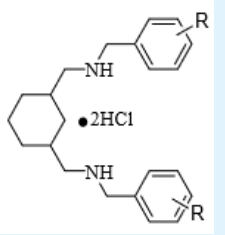

Figure 6: Prototype structure of Cyclohexane diamine derivatives as antibacterial agents.

Thiazine based heterocyclic compounds are considered as potential antimicrobial agents. Thiazine derivatives specially 1,3 and 1,4-thiazines derivatives along with along with their acylated products show strong antimicrobial activity. These compounds exhibited antibacterial activity against Staphylococcus aureus, Bacillus subtilis, Escherichia coli, Vibrio cholerae and Pseudomonas aeruginosa [26]. A class of new 3substituted indole derivatives was synthesized and evaluated for antimicrobial activities. These compounds were screened for antimicrobial activities against Staphylococcus aureus, Bacillus subtilis, Pseudomonas aeruginosa, Escherichia coli, Aspergillus fumigatus and Candida albicans. Minimum inhibitory concentration values of these derivatives were determined and gentamycin and amphotericin B were used as standard antibacterial and antifungal drugs respectively. All the tested compounds showed better antibacterial properties against gram positive bacteria as compared to gram negative bacteria, but none of compound was effective against Pseudomonas aeruginosa. N'-[2-(4-Bromophenyl)1H-indol-3-ylmethylene]-4-(1H-indol-3-yl)-

butanehydrazide was found to be best antibacterial agent while 2-\{2-[2-(4-Bromophenyl)-1H-indol-3ylmethylene]hydrazine\}-1,3-benzothiazole was found to be best antifungal agent [27]. A series of $44 \mathrm{~N}, \mathrm{~N}$-dibenzylcyclohexane-1,2-diamine derivatives were synthesized, characterized and examined for antibacterial properties against gram positive and gram negative bacteria and antifungal properties against Candida albicans, Candida glabrata and Geotrichum candidium. Minimum inhibitory concentrations of these compounds were measured and compared with Tetracycline. Many of these compounds showed antibacterial and antifungal activity. But some compounds were 37-6200 fold more active than reference compound against Candida albicans, Candida glabrata and Geotrichum candidium. Moreover all the compounds were non toxic for mammalian RBCs [28].

Newly prepared Pyrazolone derivatives called 5Pyrazolone 1,3,4-oxadiazole sulfonyl phosphonates (Figure 7) were tested for their antimicrobial properties. Disc diffusion method was used to determine the antimicrobial properties against Staphylococcus aureus, Bacillus cerus, Escherichia coli, Pseudomonas aeruginosa, Aspergillus niger and Candida albicans. These compounds showed potent antimicrobial activity against both bacteria and fungi [14].

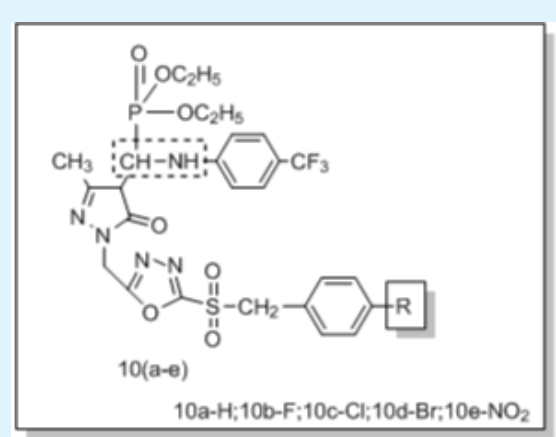

Figure 7: Structure of5-Pyrazolone 1,3,4-oxadiazole sulfonyl phosphonates.

A series of Phthalazinones derivatives were synthesized (Figure 8) and antimicrobial activity was measured using disc diffusion method. Antibacterial activity was explored against Bacillus stablius, Proteus mirabilis, Klebsiella pneumoniae, Salmonella typhi and antifungal activity was measured against Candida albicans and Aspergillus fumigatus. Phthalazinones derivative which was most potent antimicrobial agent was 2-[(4substituted phthalazin-1yl) alkyl]-1H-isoindole-1,3(2H)diones (Figure 8). It was concluded that that Phthalazinones derivatives showed better antifungal activity and their antibacterial properties were more potent against gram positive bacteria as compared to gram negative bacteria [15]. 


\section{Open Access Journal of Microbiology \& Biotechnology}

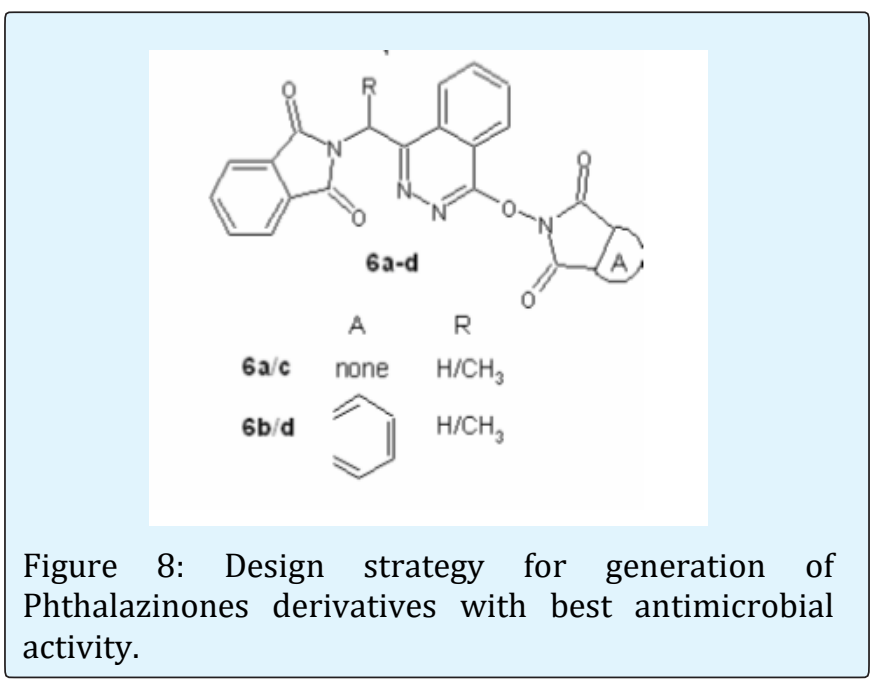

A class of 10 new 1,4-disubstituted Octahydroquinoxaline-2,3-dione derivatives were synthesized and tested for antimicrobial properties. Using agar cup diffusion method, antibacterial activity of these derivatives was determined against Serratia marscens, Pseudomonas aeruginosa, Escherichia coli, Staphylococcus aureus, Bacillus cereus and Micrococcus luteus. Antifungal properties were determined against Candida albicans, Geotrichum candidium, Fusarium oxysporum, Aspergillus flavus, Scopulariopsis brevicaulis and Trichophyton rubrum. At a concentration of $10 \mu \mathrm{mol} / \mathrm{mL}$, all the compounds showed weak to moderate antimicrobial activity in comparison to chloramphenicol and clotrimazole as reference drugs against bacteria and fungi respectively [29]. Five new derivatives of 3-allyl-1,3-thiazolidin-4-one were prepared and investigated for antimicrobial susceptibility which was performed in Mueller-Hinton agar. Minimum inhibitory concentration values were determined for Staphylococcus aureus, Enterococcus faecalis, Escherichia coli, Pseudomonas aeruginosa, Klebsiella pneumoniae, Proteus mirabilis, Bacillus subtilis, Bacillus cereus and Candida albicans. All the compounds showed antimicrobial and antifungal activity, but none of compound was found to be effective against Pseudomonas aeruginosa. Compounds containing nitro group in their structure showed better antimicrobial and antifungal activity [17]. Compounds Containing 1,2,4- Triazoleand 1,3,4-Thiadiazole rings were tested for antimicrobial properties by disc diffusion method. In vitro antimicrobial susceptibility was determined against Escherichia coli, Klebsiella pneumoniae, staphylococcus aureus and Enterococcus faecalis. All the compounds showed antibacterial and antifungal activity. One of compounds $\left(\mathrm{C}_{8} \mathrm{H}_{10} \mathrm{~N}_{10} \mathrm{~S}_{5}\right)$ showed higher antibacterial activity against Escherichia coli as compared to ceftriaxone and amoxicillin [30].

Different isatin derivatives (6 compounds) were synthesized (Figure 9) and their antimicrobial profile was determined against Staphylococcus aureus, Listeria monocytogenes, Enterococcus faecalis, Shigella sonnei, Salmonella enteritidis, Yersinia enterocolitica, Escherichia coli, Proteus hauseri, Pseudomonas aeruginosa and Candida albicans. The broth micro-dilution method was used to measure the minimum inhibitory concentration of compounds. All the six derivatives showed substantial antimicrobial activity except compound (1,3-dihydro-3[(4-cyanophenyl) imino]-2H-indol-2-one). The most potent antimicrobial compound was found to be 1,3dihydro-3-[(5-mercapto-1,3,4-thiadiazol-2-yl)imino]-2H-indol-2-one. All the isatin derivatives showed better antibacterial activity against gram positive bacteria as compared to gram negative bacteria [18].

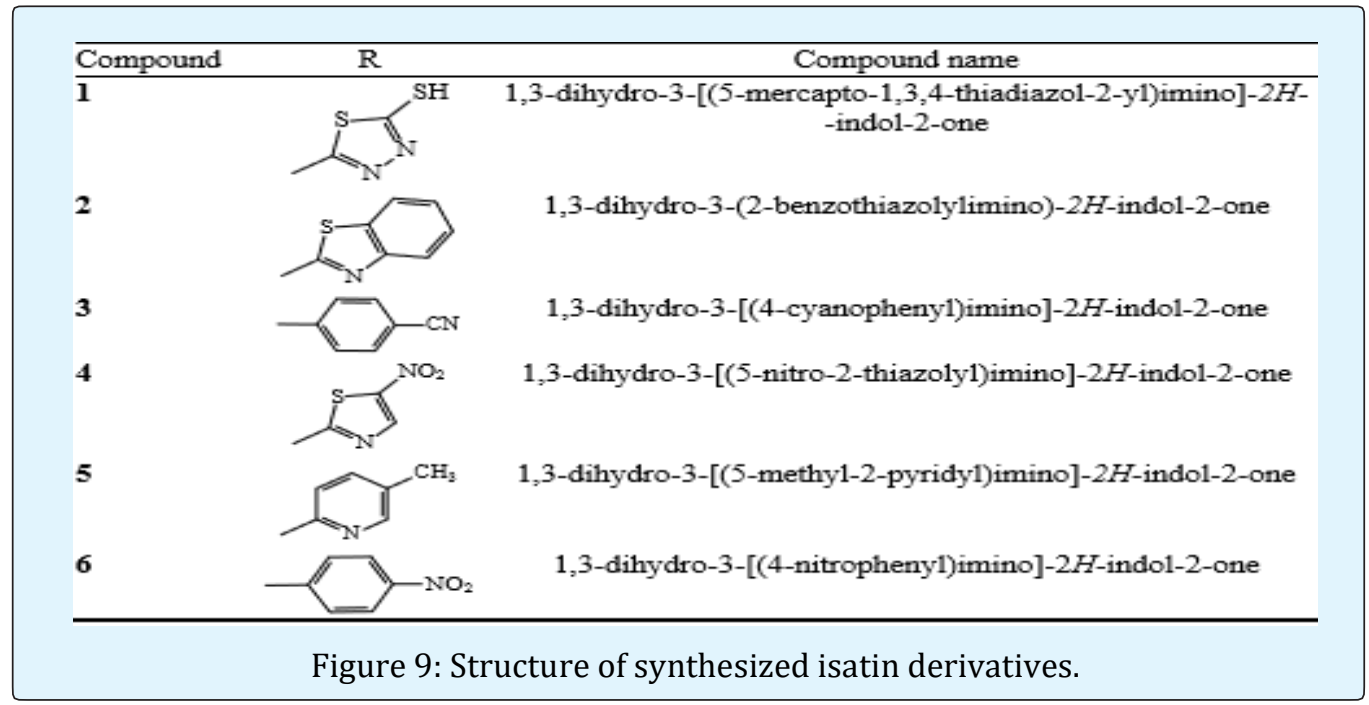

Shoaib M and Ganbarov G Kh. Functionally Substituted Chemical Organic Compounds: Potential Antimicrobial Substances. J Microbiol Biotechnol, 2019, 4(1): 000136. 


\section{Open Access Journal of Microbiology \& Biotechnology}

Antifungal activity of 12 organic compounds derived from amino alcohols was measured. These compounds were synthesized from p-hydroxybenzaldehyde and $\mathrm{p}$ hydroxybenzoic acid. In vitro antifungal activity was measured against Trichophyton rubrum, Trichophyton mentagrophytes and Candida albicans. Minimum inhibitory concentrations were determined and it was noted that amine series was found to be more effective against filamentous fungi and yeast. The amide series derivatives showed no considerable antifungal effects [31]. Five thiourea derivatives (Figure 10) were prepared and were tested for antibacterial and anti yeast activity. The minimum inhibitory concentration was determined for bacteria (Staphylococcus aureus, Staphylococcus epidermidis, Enterococcus faecalis, Streptococcus pyogenes, Bacillus cereus, Esherichia coli, Pseudomonas aeruginosa, Enterobacter cloacae, Proteus vulgaris, and Enterobacter aerogenes) and yeast (Candida albicans, Candida krusei, Candida glabrata, Candida tropicalis, and Candida parapsilosis). All the tested thiourea derivatives showed high antibacterial and low anti yeast activity [32].

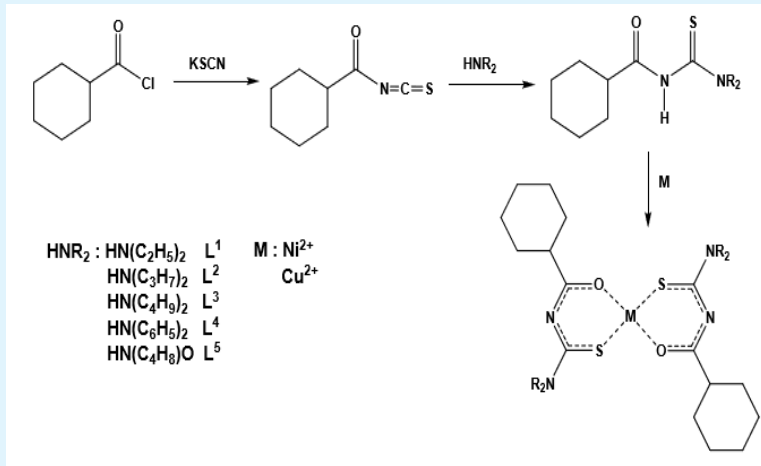

Figure 10: Synthesis of thiourea derivatives.

A new series of chalcones, 3-(2-hydroxy-5-(aryldiazenyl)phenyl)-1-(aryl)prop-2-en-1-one and their pyrimidine derivatives i.e. 4-(2-hydroxy-5(aryldiazenyl)phenyl)-6-(aryl)pyrimidin-2-ols were synthesized and investigated for antimicrobial properties. Muller Hinton agar was used to determine antibacterial properties against Staphylococcus aureus, Bacillus subtilis, Proteus vulgaris and Escherichia coli. Dextrose agar was used to determine antifungal properties against Candida albicans and Aspergillus niger. All the compounds showed considerable antimicrobial activity but it was concluded that pyrimidine derivatives having nitro group in their structure showed better antimicrobial properties as compared to other chalcones derivatives [33]. Thiocarbohydrazide derived new heterocyclic Schiff bases were prepared and evaluated for antimicrobial properties against Staphylococcus aureus, Bacillus subtilis, Salmonella typhimurium, Escherichia coli and Candida albicans by disc agar diffusion method. Compounds having formula 3$\{\quad\{[\quad[(5,6-$ dioxo-3-thioxo-1,2,4triazinan-4yl)imino] ]methyl\}\}-6,7,8,9tetrahydropyrazolo[ [1,5a] ][ [1] ]benzothieno [ [2,3-d] ]pyrimidin-10(4H)-one and $3-\{\{[$ [(6-methyl-5-oxo-3-thioxo2,5-dihydro-1,2,4-triazin4(3H)-yl)imino] ] methyl\} \}-6,7,8,9tetrahydropyrazolo[ [1,5-a] ] [ [1] ]benzothieno[ [2,3d] ]pyrimidin-10 $(4 \mathrm{H})$ one exhibited maximum antimicrobial activity [34]. 2-Styrylchromones are novel class of organic compounds which are structurally related to flavones. Antimicrobial activity of three new 2styrylchromones and their analogues was determined by agar cup method against Xanthomonas campesrtis and Agrobacterium tumafeciens. Antifungal activity was determined against Aspergillus niger and Penicillium chrysogenum by using disc diffusion method. All the synthesized compounds showed antimicrobial activity, but hydroxy substituted compounds were better antimicrobial agents [35]. The compound N-nitroso-2,6diphenylpiperidin-4-one semicarbazone was prepared and analyzed for its antimicrobial properties. Antimicrobial screening was done against Bacillus subtilis, Staphylococcus aureus, Escherichia coli and Candida albicans by using disc diffusion method. The compound exhibited good antifungal and moderate antibacterial activity. High hydrophobic content and piperidine ring system contribute to high antimicrobial activity [36].

\section{Halogenated Compound Possessing Antimicrobial Activity}

Hydroxylactone, obtained by biotransformation of Bromo and Iodolactone with Gem-Dimethylcyclohexane ring, was examined for antimicrobial activity against bacteria, fungi and yeast. Hydroxylactone completely inhibited the growth of Micrococcus flavus, Pseudomonas fluorescens and Alternaria species and retarded the growth of Bacillus cereus, Debaryomyces hansenii and Penicillium species. Hydroxylactone showed no effect on growth of Escherichia coli, Bacillus subtilis, Saccharomyces cerevisiae, Yarrowia lipolytica, Schizosaccharomyces pombe, Rhodotorula rubra and Aspergillus niger [37]. Pinicoloform is an unbranched acyclic compound which contains a trichloromethyl group. This compound was isolated from extracts of the mycelia of the Basidiomycete Resinicium pinicola. In the serial dilution assay, Pinicoloform has shown unselective antimicrobial activity against different bacteria and fungi including Acinetobacter calcoaceticus, Corynebacterium insidiosum, Mycobacterium phlei, Paecilomyces variotii and different 


\section{Open Access Journal of Microbiology \& Biotechnology}

strains of Saccharomyces cerevisiae [38]. Quaternary ammonium compounds bearing mixed $\mathrm{N}$-substituted groups called quaternary ammonium bromides (Figure 11) were tested for screened for antimicrobial activity against Staphylococcus aureus, Pseudomonas aeruginosa and Shigella. Quaternary ammonium bromides showed strong activity against Staphylococcus aureus and weak antimicrobial activity against Shigella. Pseudomonas aeruginosa was found to be resistant against quaternary ammonium bromides [7].

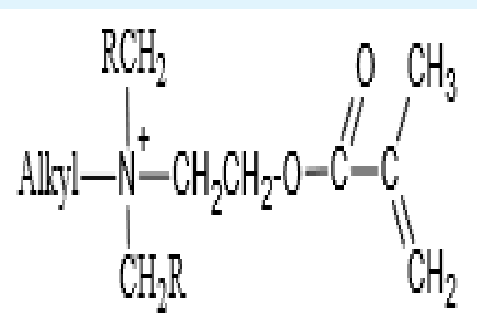

Figure 11: Structural formula of quaternary ammonium bromides.
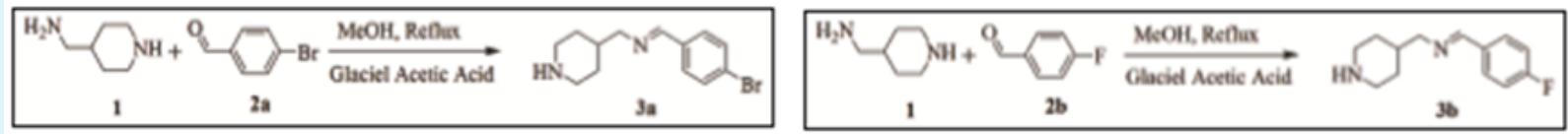

Figure 12: Preparation of Schiff bases of piperidine containing Bromine and Fluorine group.

Two Schiff bases derived from 4-(aminomethyl) piperidine having structural formula $\mathrm{N}-(4-$ bromobenzylidene)(piperidin4-yl)methanamine and $\mathrm{N}$ (4-fluorobenzylidene)(piperidin4-yl)methanamine (Figure 12) were synthesized. These compounds were tested for antimicrobial properties against Escherichia coli, Pseudomonas aeruginosa and Staphylococcus aureus using agar well diffusion method. The compounds containing flourobenzylidene showed strong antibacterial activity as compared to compound containing bromobenzylidene [39].

A series of fluorinated $10 \mathrm{H}$-Phenothiazines and their sulfone derivatives were synthesized and screened for antimicrobial activity. Kerby Bauer procedure by using Streptomycin as reference antibacterial compound was used to determine antimicrobial properties of these compounds against Staphylococcus aureus, Pseudomonas flueroscence, Aspergillus flavus and Aspergillus niger. All the tested compounds showed remarkable antimicrobial profile [40]. Several halogens substituted tricyclic sulfur containing flavonoids (Figure 13) were prepared and analyzed for antibacterial properties against Staphylococcus aureus and Escherichia coli. The effect of different halogen substitution on antimicrobial profile of these compounds was noted. It was concluded that by introduction of any halogen except Fluorine in the structure of tricyclic flavonoids improved the antibacterial activities against both gram positive and gram negative bacteria [41].

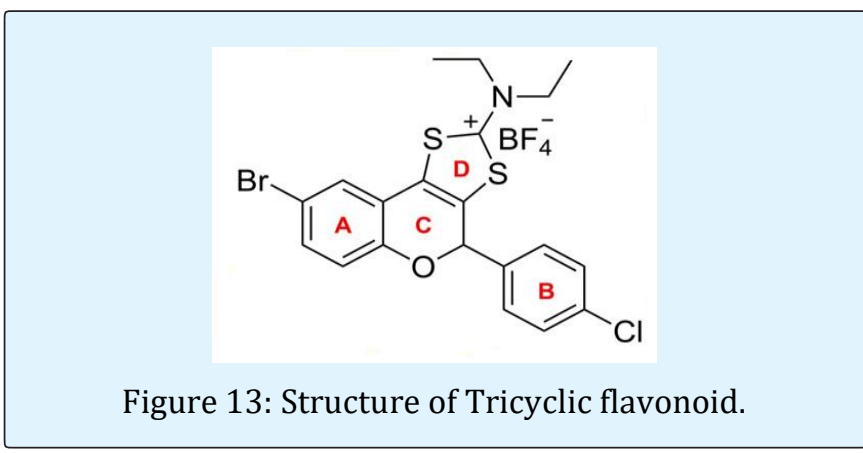

\section{Conclusion}

Antimicrobial resistance is a serious global health problem due to ever increasing number of drug resistant, multi drug resistant, extensively drug resistant and pan drug resistant microorganisms. This perpetual increasing antimicrobial resistance is major obstacle in control of infectious diseases worldwide. To ameliorate this condition, globally numerous efforts are being made and new drug development studies are being conducted. In this regard, variety of chemical organic compounds and their new functionally substituted derivatives discussed in review are best available option for candidate antimicrobial drugs in the future. Numerous organic compounds with subtle substitutions in their structure have exhibited potential antimicrobial profile. It is need of hour to expedite these efforts to develop new antimicrobial drugs. 


\section{Open Access Journal of Microbiology \& Biotechnology}

\section{References}

1. Vnutskikh ZA, Shklyaev YV, Odegova TF, Chekryshkin, YS, Tolstikov AG, et al. (2006) Synthesis and antimicrobial activity of mono-and biquaternized derivates of dipyridylethanes and dipyridylethylenes. Pharm Chem J 40(4): 194-198.

2. Kumar D, Rajesh KR, Nilanjan R, Diwan SR (2011) Synthesis and Antibacterial Activity Evaluation of Unsymmetrically Substituted Cyclohexane-1,2diamine Derivatives. Chem \& Bio Inter 1(2): 263-278.

3. Bielawski K, Katarzyna L, Zbigniew K, Anna B, Olga M, et al. (2017) Synthesis and antimicrobial activity of chiral quaternary $\mathrm{N}$-spiro ammonium bromides with 3',4'-dihydro-1' $\mathrm{h}$-spiro[isoindoline-2,2' isoquinoline] skeleton. Drug Des Devel Ther 11: 2015-2028.

4. Tsemeugne J, Emmanuel SF, Jean-de-Dieu T, Taoufik R, Arnaud DN, et al. (2018) Synthesis, Characterization, and Antimicrobial Activity of a Novel Trisazo Dye from 3-Amino-4H-thieno[3,4c][1]benzopyran-4-one. Int J Med Chem 2: 1-8.

5. Alptüzün V, Sülünay P, Hüseyin T, Ercin E (2009) Synthesis and Antimicrobial Activity of Some Pyridinium Salts. Molecules 14(12): 5203-5215.

6. Zhao T, Sun GJ (2008) Hydrophobicity and antimicrobial activities of quaternary pyridinium salts. J Appl Microbiol 104(3): 824-830.

7. Daoudi S, Adil AO, Tahar B, Zahra OK (2014) Synthesis, characterization and antibacterial activity of Quaternary Ammonium Compounds bearing mixed N-substituted groups. Chem Sci Trans 3(1): 281-291.

8. Batanony NH (2017) Antimicrobial activities and mode of action of the selected novel thienopyrimidines derivatives 2-[2(diphenylmethylene) hydrazino]-5-isopropyl-3methylthieno [2, 3-d] pyrimidin-4-one. Period Biol 119(1): 27-36.

9. Sabbineni J (2016) Phenol-An effective antibacterial Agent. JOMC 3(2): 182-191.

10. Babii C, Bahrin LG, Neagu AN, Gostin I, Mihasan M, et al. (2016) Antibacterial activity and proposed action mechanism of a new class of synthetic tricyclic flavonoids. J Appl Microbiol 120(3): 630-637.
11. Masunari A, Tavares LC (2007) A new class of nifuroxazide analogues: Synthesis of 5nitrothiophene derivatives with antimicrobial activity against multidrug-resistant Staphylococcus aureus. Bioorg Med Chem 15(12): 4229-4236.

12. Al-Refai M, Ibrahim M, Al-Fawwaz A, Geyer A (2017) One-pot synthesis and antimicrobial activity of new 4,6-disubstituted-3,4-dihydropyrimidine-2(1H)thiones. Chem Eur J 8(1): 96-100.

13. Lloyd WJ, Broadhurst AV, Hall MJ, Andrews KM, Barber WE, et al. (1988) Cyclohexane Triones, Novel Membrane-Active Antibacterial Agents. Antimicrob Agents Chemother 32(6): 814-818.

14. Rani VE, Ravindranath LK (2016) Synthesis and Antimicrobial Activity of Novel Pyrazole-5-one Containing 1, 3, 4-oxadiazole Sulfonyl Phosphonates. J Org Chem 6(1): 1-7.

15. Salvi VK, Bhambi D, Jat JL, Talesara GL (2006) Synthesis and antimicrobial activity of some 2-[1-(4oxo-3,4dihydrophthalazine-1-yl)alkyl]-1H-isoindole$1,3(2 \mathrm{H})$-dione and their imidoxy derivatives. ARKIVOC 14: 133-140.

16. Petrikaitè V, Tarasevičius E, Pavilonis A (2007) New ethacridine derivatives as the potential antifungal and antibacterial preparations. Medicina 43(8): 657-663.

17. Ževžikovienė A, Ževžikovas A, Tarasevičius E, Šlepikas L, Vitkevičius K (2011) Synthesis and Antimicrobial Activity of New 3-Allyl-1,3-Thiazolidin4-Ones. Medicina 47(2): 78-84.

18. Šekularac GM, Nikolić JB, Petrović P, Bugarski B, Đurović B, et al. (2014) Synthesis, antimicrobial and antioxidative activity of some new isatin derivatives. J Serb Chem Soc 79(11): 1347-1354.

19. Beena Kumar D, Kumbukgolla W, Jayaweera S, Bailey M, Alling T, et al. (2014) Antibacterial activity of adamantyl substituted cyclohexane diamine derivatives against methicillin resistant Staphylococcus aureus and Mycobacterium tuberculosis. RSC Advances 4(23): 11962-11966.

20. Zarafu I, Turcu I, Culiță DC, Petrescu S, Popa M, et al. (2018) Antimicrobial Features of Organic Functionalized Graphene-Oxide with Selected Amines. Materials 11(9): 1704-1713. 


\section{Open Access Journal of Microbiology \& Biotechnology}

21. Modiya PR, Patel CN (2012) Synthesis and screening of antibacterial and antifungal activity of 5-chloro1,3-benzoxazol-2(3 h)-one derivatives. Org Med Chem Lett 2(1): 29-38.

22. Lubenets V, Vasylyuk S, Monka N, Bolibrukh K, Komarovska-Porokhnyavets 0, et al. (2016) Synthesis and antimicrobial properties of 4acylaminobenzenethiosulfoacid S-esters. Saudi Pharm J 25(2): 266-274.

23. Reddy CUM, Jayakar B, Srinivasan R (2010) Synthesis and antimicrobial activity of $\alpha \mathrm{N}$-phthilimido and acetimido derivatives from amino acids and anhydrides. Int J Pharma Bio Sci 1(4): 81-86.

24. Salman AS, Abdel-Aziem A, Alkubbat MJS (2015) Synthesis, Spectroscopic Characterization and Antimicrobial Activity of Some New 2-Substituted Imidazole Derivatives. IJOC 5(1): 15-28.

25. Becenen N, Uluçam G, Altun O (2017) Synthesis and antimicrobial activity of iron cyclohexanedicarboxylic acid and examination of ph effect on extraction in water and organic phases. Trak univ Journal of Nat Sci 18(1): 1-7.

26. Sharma PK (2017) A review: antimicrobial agents based on nitrogen and Sulphur containing heterocycles. Asian J Pharm Clin Res 10(2): 47-49.

27. Salman AS, Mahmoud NA, Abdel-Aziem A, Mohamed MA, Elsisi DM (2015) Synthesis, Reactions and Antimicrobial Activity of Some New 3-Substituted Indole Derivatives. IJOC 5(2): 81-99.

28. Sharma M, Joshi P, Kumar N, Joshi S, Rohilla RK, et al. (2011) Synthesis, antimicrobial activity and structure-activity relationship study of N,N-dibenzylcyclohexane-1,2-diamine derivatives. Eur J Med Chem 46(2): 480-487.

29. Hussein MA (2011) Design, Synthesis, and Antimicrobial Activity, of New 1,4-disubstituted Octahydroquinoxaline-2,3-dione Derivatives. Bull Korean Chem Soc 32(5): 1511-1518.

30. Abdulrasool MM, Jawad AH, Shneine JK (2012) Synthesis, Characterization and Evaluation of Biological Activity of New Heterocyclic Compounds Containing 1,2,4- Triazoleand 1,3,4-Thiadiazole Rings. IJAST 2(10): 155-164.
31. Caneschi CA, Almeida AM, Martins FJ, Hyaric ML, Oliveira MME, et al. (2017) In vitro antifungal activity of organic compounds derived from amino alcohols against onychomycosis. Braz J Microbiol 48(3): 476482.

32. Arslan H, Duran N, Borekci G, Koray C, Akbay C (2009) Antimicrobial Activity of Some Thiourea Derivatives and Their Nickel and Copper Complexes. Molecules 14(1): 519-527.

33. Pandhurnekar CP, Meshram EM, Chopde HN, Batra RJ (2013) Synthesis, Characterization, and Biological Activity of 4-(2-Hydroxy-5-(aryl-diazenyl)phenyl)-6(aryl)pyrimidin-2-ols Derivatives. Org Chem Int 1-10.

34. El-Mahdy KM, El-Kazak AM, Abdel-Megid M, Seada M, Farouk 0 (2016) Synthesis, Characterization and Antimicrobial Activities of Some New Heterocyclic Schiff Bases Derived from Thiocarbohydrazide. Acta Chim Slov 63(1): 18-25.

35. Madhava RV, Ujwala B, Priyadarshini P, Krishna MP (2016) Synthesis, antioxidant and antimicrobial activity of three new 2-styrylchromones and their analogues. Der Pharma Chemica 8(7): 1-6.

36. Mubarak S, Sirajudheen P, Shebin KSM, Muhasina M, Rishana T (2015) Synthesis Characterization and Antimicrobial Activity of N-nitroso-2, 6Diphenylpiperidin-4-One Semicarbazone. Organic Chem Curr Res 4(2): 141-146.

37. Grabarczyk M, Mączka W, Wińska K, Żarowskab B, Anioła MJ (2013) Antimicrobial Activity of Hydroxylactone obtained by Biotransformation of Bromo- and Iodolactone with GemDimethylcyclohexane Ring. Braz Chem Soc 24(12): 1913-1919.

38. Becker U, Anke T, Sterner O (1994) A Novel Halogenated Compound Possessing Antibiotic and Cytotoxic Activities Isolated from the Fungus Resinicium pinicola Erikss \& Hjortst Z Naturforsch 49(c): 772-774.

39. Karthik CS, Mallesha L, Santhosh MV, Mallu P (2016) Synthesis, Characterization, Antimicrobial Activity, and Optical Properties of Schiff Bases Derived from 4(Aminomethyl) Piperidine. Indian J Adv Chem Sci S1: 206-212. 
40. Ahmad I, Shagufta (2015) Sulfones: An important class of organic compounds with diverse biological activities. Int J Pharm Sci 7(3): 19-27.
41. Bahrin LG, Hopf H, Jones PG, Sarbu LG, Babii C, et al. (2016) Antibacterial structure-activity relationship studies of several tricyclic sulfur-containing flavonoids. Beilstein J Org Chem 12: 1065-1071. 